\section{Colored Plastic Mulches Influence Cucumber Beetle Populations, Vine Growth, and Yield of Watermelon}

\author{
J.R. Andino and C.E. Motsenbocker \\ Department of Horticulture, Louisiana State University Agricultural Center, \\ 137 J.C. Miller Hall, Baton Rouge, LA 70803
}

Additional index words. Citrullus lanatus, wavelength selective mulch, reflective mulch, triploid watermelon

\begin{abstract}
Colored plastic mulches were evaluated for their effect on the production of a triploid ('Honeyheart') and a diploid ('Sangria') watermelon cultivar during the spring growing season. Colored mulches affected cucumber beetle populations; the SRM-Red (Selective Reflective Mulch) and yellow plastic mulch plots had among the highest cucumber beetle populations recorded in both cultivars while the silver-reflective and the silver-on-black plastic mulches had among the lowest. In general, most mulched plots had longer vines than the bare-ground treatment, with few differences in vine length among treatments by 4 weeks. There were no differences among mulch treatments in first and total 'Honeyheart' harvest while the IRT-100 (infrared transmitting; green), PST (photosynthetic reduced transmitting), and silver-on-black plastic mulches had the highest first 'Sangria' harvest and among the highest total 'Sangria' harvest. Plants in plastic mulch treatments had higher yields as a result of higher fruit number per area. Fruit weight, length, and diameter and total soluble solids for both cultivars were not affected by colored plastic mulch treatments.
\end{abstract}

Reflective and wavelength-selective plastic mulches (colored mulches), such as silver reflective and IRT-100 (infrared-transmitting; green) or SRM-red (selective reflective mulchred) mulches, respectively, are mulch materials that are increasingly being used commercially. Colored mulches have advantages similar to black or clear plastic mulches with additional benefits related to altered quantity and quality of reflected light into the plant canopy. The effects of colored plastic mulches on plant growth and yield have been studied in a number of vegetable crops such as bell pepper (Capsicum annuum L.) (Decoteau et al., 1990), cowpea (Vigna unguiculata L.) (Hunt et al., 1990), muskmelon (Cucumis melo L.) (Taber, 1993), tomato (Lycopersicon esculentum Mill.) (Decoteau et al., 1989), and potato (Solanum tuberosum L.) (Matheny et al., 1992).

Previous field research with black and/or white plastic mulches indicated higher watermelon yield compared to bare ground culture (Brinen et al., 1979; Sanders et al., 1999; Soltani et al., 1995). Very limited research has been conducted to evaluate watermelon crop response to colored plastic mulches in the field. The effect of light quality on the growth of young watermelon plants in controlled environments was evaluated previously (Decoteau and Friend, 1991; Hatt and Decoteau, 1997; Decoteau and Hatt, 1997). Watermelon plants responded to

Received for publication 12 July 2003 . Accepted for publication 3 Nov. 2003. From a thesis submitted by J.Andino in partial fulfillment of the requirements for a MS degree. Mention of a trade mark, proprietary product, or vendor does not constitute a guarantee or warranty of the product by the LSU AgCenter and does not imply its approval to the exclusion of other products or vendors that also may be suitable. light environment changes (high far red to red light ratio exposure; FR/R) by partitionresulting in plants with elongated leaf petioles and internodes. Researchers demonstrated that vegetative plant growth can be directly affected by the quality of radiation reflected from certain mulches (Decoteau et al., 1989 and Decoteau et al., 1990). Even though the effect of colored plastic mulches on watermelon is temporal, until the vines of the crop cover the mulch, there is the hypothesis that early plant response to the light environment can induce modifications in the plant growth that continue after the vines cover the plastic.

The objective of this experiment was to determine the effect of different reflective and wavelength selective plastic mulches on insect populations, plant growth, and yield of fieldgrown watermelon.

\section{Materials and Methods}

Field experiments were conducted during the Spring 1997 and 1998 at the Burden Research Center, Baton Rouge, La. Plots received the equivalent in $\mathrm{kg} \cdot \mathrm{ha}^{-1}$ of $41 \mathrm{~N}-121 \mathrm{P}-121 \mathrm{~K}$ based on soil sampling and testing following Louisiana Cooperative Extension Service recommendations (Boudreaux, 1996). Raised beds $15 \mathrm{~cm}$ high $\times 60 \mathrm{~cm}$ wide and $2.4 \mathrm{~m}$ from center to center were used. Trickle irrigation tape (Turbulent Twin-Wall, Chapin Watermatics, Watertown, N.Y.) was installed $10-\mathrm{cm}$ off center at a $7-\mathrm{cm}$ depth. Irrigation was applied when soil tensiometer readings $(15 \mathrm{~cm})$ reached $-20 \mathrm{kPa}$ and terminated at $-10 \mathrm{kPa}$.

Plastic mulch treatments (Table 1) were installed using a plastic mulch layer machine on 3 Apr. 1997, and 23 Mar. 1998. Painted mulch ing more photosynthate to vegetative parts, treatments, blue in 1997 and 1998 and yellow in 1997, were established by application of oil base paint (Benjamin Moore and Company, Montvale, N.J.) diluted 2:1 (v/v) with thinner and applied with a back-pack sprayer over black plastic mulch. Because a commercial yellow plastic mulch was not available in 1997, a yellow-painted mulch treatment was used. A commercial yellow mulch, however, was used in 1998. Bare ground and black plastic mulch were used as controls. A yellow-flesh triploid, commonly called seedless, ('Honeyheart'; Seminis, Saticoy, Calif.) and a red-flesh diploid (seeded) cultivar ('Sangria', Syngenta, Boise, Idaho) were planted in 72-cell trays (Speedling planter flat, Hummert International, Earth City, Mississippi) on 13 Mar. 1997 and 9 Mar. 1998, and grown in a greenhouse set to $27 / 21^{\circ} \mathrm{C}$ day/night, respectively. Transplants were hand planted on the center of the bed to a $1.5-\mathrm{m}$ in-row spacing (six plants per plot) on 17 Apr. 1997 and 15 Apr. 1998 based on Louisiana Cooperative Extension Service recommendations (Boudreaux, 1996). The soil was an Olivier silt loam (Typic Paleudults). The main plots (mulch treatments) consisted of four rows $9.0 \mathrm{mlong}$ with the two cultivars (subplots), 'Honeyheart' and 'Sangria', randomly assigned to the two middle rows.

Main plots were arranged in a randomized complete block design with four replications. 'Crimson Sweet' (Seminis) was used on the two outside guard rows, and together with 'Sangria', served as source of pollen for 'Honeyheart'. During the growing season, weekly fertigations of $\mathrm{NH}_{4} \mathrm{NO}_{3}$ supplied a total of $86 \mathrm{~kg} \cdot \mathrm{ha}^{-1}$ of N. Fertigations were scheduled based on the procedure described by Hochmuth (1992); $70 \%$ of the total $\mathrm{N}$ was applied during the first 5 weeks after transplanting and the remaining was applied up to week 8 after transplanting. Beehives were placed in the field $30 \mathrm{~d}$ after transplanting to provide for pollination. Insect, disease, and weed management practices were conducted in accordance with Louisiana Cooperative Extension Service recommendations (Boudreaux, 1996).

Insect populations were recorded by counting spotted cucumber beetle (Diabrotica balteata howardi (Barber)) and stripped cucumber beetle (Acalymma vittata (Fabricius)), the principal insect pests both years, on four plants per plot, 2 and 4 weeks after transplanting. After each count insects were controlled by application of Sevin (1-naphthyl-methyl carbamate) at $0.6 \mathrm{~kg}$ ai/ha. Main vine length was measured, on the four center plants per plot, from the cotyledons to the tip of the main vine 3 and 4 weeks after transplanting in 1997 and 2 and 4 weeks after transplanting in 1998. Harvest began on 7 July 1997, and 22 June 1998 with two subsequent harvests 1 week apart each. Only the first harvest was considered early yield. Atharvest, fruit from each plot were counted, measured, and weighed individually and then classified as marketable or cull fruit for separate analysis. 'Honeyheart' fruit weighing $<3.6 \mathrm{~kg}$ and 'Sangria' fruit weighing $<4.6 \mathrm{~kg}$ and misshapen or diseased fruit were considered culls. Total soluble solids were determined from two fruit of each plot at the first harvest using a hand-held refractometer. Soil 
and air temperatures for each mulch treatment were recorded using a data logger (Campbell Scientific, Logan, Utah). The data logger was programmed to monitor temperatures each hour and output the maximum and minimum temperatures with times of occurrence, every $24 \mathrm{~h}$. Temperature data were recorded daily for 49 d (7 weeks) each year, 19 Apr. to 6 June 1997, and 14 Apr. to 2 June 1998 with one repetition per treatment. Copper-constantan double insulated thermocouples were used to measure temperatures $5-\mathrm{cm}$ below the mulch surface both years. Thermocouples were located between the two center plants of one of the two subplots' rows, 15 -cm off center of the bed on the opposite side of the trickle irrigation tape position. Daily temperature averages (49 repetitions) were used for repeated measures analysis using SAS Mixed Model Procedure and means separated by Fisher's protected least significant difference (LSD) at the 0.05 significance level (SAS Institute, Cary, N.C.). Data on beetle populations, plant growth, and yield were subjected to an analysis of variance using SAS Mixed Model procedure and means separated by Fisher's protected LSD at the 0.05 significance level (SAS Institute, Cary, N.C.).

\section{Results and Discussion}

Insect populations. Mulch treatments affected spotted and stripped cucumber beetle both years (Table 2). The highest beetle populations were counted, at both dates, in 'Honeyheart' on the SRM-Red and yellow plastic mulch plots; at 2 weeks after transplanting these populations were similar to PST mulch and at 4 weeks after transplanting to Al-Or brown, black, IRT-100, and PST mulches. The lowest beetle populations in 'Honeyheart' both dates ( $<1$ beetle per plant) were present in the silver-reflective and silveron-black plastic mulch plots; however, these populations were statistically different only from the SRM-Red and yellow mulches. In 'Sangria' plants at 2 weeks after transplanting, the SRM-Red, PST, and yellow mulches had the highest cucumber beetle numbers, similar to Al-Or Brown. At 4 weeks after transplanting, the highest populations were counted on SRM-Red, which was similar to PST. In 'Sangria' the lowest populations both dates were observed on the silver-reflective mulch; beetle numbers were statistically different from PST, SRM-Red, and yellow mulches at 2 weeks after transplanting and SRM-Red mulch at 4 weeks after transplanting.

Silver-reflective plastic mulch has been reported by several researchers to repel various species of insects, including cucumber beetles (Brown et al., 1993; Lamont et al., 1990; Schalk et al., 1979; Schalk and Robbins, 1987). Silver-reflective plastic mulch has been associated with higher light reflectance, causing insect disorientation (Zalom and Cranshaw,
1981). It was observed that plants grown in bare ground plots were smaller, which may partially explain the lower cucumber beetle populations in these plots.

Vine length. In 1997 mulch treatments produced longer vines than bare ground treatment, with the exception of 'Honeyheart' on white-on-black and yellow plastic mulches with similar vine length to the bare ground treatment at 4 weeks after transplanting (Table 3). Three weeks after transplanting, the black plastic mulch treatment had longer 'Honeyheart' main vines than Al-Or brown, silver reflective, SRM-Red, white-on-black and yellow mulch treatments; however, they were similar in length to silver-on-black, PST, IRT-100 and blue-painted plastic mulches. 'Sangria' vine lengths in 1997 were similar for all the mulch treatments except for shorter vines for yellow mulch at 3 weeks. At 4 weeks after transplanting there were no differences in vine length among mulch treatments.

Two weeks after transplanting in 1998 the vine length on Al-Or brown, black, bluepainted, IRT-100, PST and SRM-Red mulch treated plots were longer than vines in the bare ground plot for both cultivars (Table 3 ). At 4 weeks after transplanting, all the mulch treatments resulted in longer vines than the bare ground treatment. Moreover, the silverreflective, silver-on-black and SRM-Red mulch treatments had longer 'Honeyheart' vines than black plastic mulch plots. There were no differ-

Table 1. Treatments evaluated for air and soil temperature, plant growth, insect populations and watermelon yield, 1997 and 1998.

\begin{tabular}{|c|c|c|}
\hline Mulch $^{2}$ & Color/type & Company \\
\hline$\overline{\mathrm{Al}-O r}$ brown (aluminum-organic) & Brown/wavelength selective & Polywest, San Diego, Calif. \\
\hline Black & Black & Climagro, Quebec, Canada \\
\hline Bare ground & --- & --- \\
\hline Blue-painted black & Blue & --- \\
\hline IRT-100 (infrared transmitting) & Green/wavelength selective & Ken-Bar, Reading, Mass. \\
\hline PST (photosynthetic reduced transmitting) & Green/wavelength selective & Climagro, Quebec, Canada \\
\hline Silver-reflective & Silver/reflective & Clarke Ag. Plastics, Greenwood, Va. \\
\hline Silver-on-black & Silver/reflective & Climagro, Quebec, Canada \\
\hline SRM-Red (selective reflective mulch) & Red/wavelength selective & Ken-Bar, Reading, Mass. \\
\hline White-on-black & White/reflective & Climagro, Quebec, Canada \\
\hline Yellow & Yellow & ---/Polywest, San Diego, Calif. \\
\hline
\end{tabular}

${ }^{2}$ All plastic mulch treatments thickness was 1 mil, except for Al-Or brown, 0.8 mil, silver-reflective, 0.9 mil, and yellow-Polywest, 1.25 mil.

${ }^{y}$ Yellow-painted black mulch in 1997 and yellow on brown mulch (Polywest) in 1998.

Table 2. Effect of colored plastic mulches on soil temperature and cucumber beetles.

\begin{tabular}{|c|c|c|c|c|c|c|c|c|}
\hline \multirow[b]{4}{*}{ Treatment } & \multirow{2}{*}{\multicolumn{4}{|c|}{ Soil temp ${ }^{z}\left({ }^{\circ} \mathrm{C}\right)$}} & \multicolumn{4}{|c|}{ Cucumber beetles (no. per plot) ${ }^{y}$} \\
\hline & & & & & \multicolumn{2}{|c|}{ Honeyheart } & \multicolumn{2}{|c|}{ Sangria } \\
\hline & \multicolumn{2}{|c|}{ Min } & \multicolumn{2}{|c|}{ Max } & \multicolumn{4}{|c|}{ Weeks after transplanting } \\
\hline & 1997 & 1998 & 1997 & 1998 & 2 & 4 & 2 & 4 \\
\hline Al-Or brown ${ }^{x}$ & $19.4 \mathrm{~cd}^{\mathrm{w}}$ & $21.7 \mathrm{c}$ & $37.1 \mathrm{c}$ & $36.0 \mathrm{~d}$ & $2.0 \mathrm{~b}$ & $6.7 \mathrm{ab}$ & $1.9 \mathrm{ab}$ & $6.9 \mathrm{~b}$ \\
\hline Bare ground & $18.5 \mathrm{e}$ & $19.6 \mathrm{~g}$ & $29.0 \mathrm{~g}$ & $30.7 \mathrm{~g}$ & $0.7 \mathrm{~b}$ & $3.2 \mathrm{~b}$ & $0.6 \mathrm{~b}$ & $3.1 \mathrm{~b}$ \\
\hline Black & $19.8 \mathrm{bc}$ & $21.4 \mathrm{~d}$ & $39.2 \mathrm{~b}$ & $38.3 \mathrm{~b}$ & $1.0 \mathrm{~b}$ & $11.1 \mathrm{ab}$ & $1.2 \mathrm{~b}$ & $6.2 \mathrm{~b}$ \\
\hline Blue-painted & $19.9 \mathrm{~b}$ & $21.5 \mathrm{~cd}$ & $36.4 \mathrm{~cd}$ & $35.4 \mathrm{~d}$ & $0.5 \mathrm{~b}$ & $4.2 \mathrm{~b}$ & $0.5 \mathrm{~b}$ & $2.9 \mathrm{~b}$ \\
\hline IRT-100 & $19.9 \mathrm{~b}$ & $21.6 \mathrm{c}$ & $40.7 \mathrm{a}$ & $39.5 \mathrm{a}$ & $1.7 \mathrm{~b}$ & $7.8 \mathrm{ab}$ & $0.6 \mathrm{~b}$ & $8.1 \mathrm{~b}$ \\
\hline PST & $19.8 \mathrm{bc}$ & $22.2 \mathrm{~b}$ & $36.4 \mathrm{~cd}$ & $38.1 \mathrm{bc}$ & $3.7 \mathrm{ab}$ & $10.7 \mathrm{ab}$ & $5.1 \mathrm{a}$ & $21.5 \mathrm{ab}$ \\
\hline Silver-reflective & $20.5 \mathrm{a}$ & $22.5 \mathrm{a}$ & $33.6 \mathrm{e}$ & $30.4 \mathrm{~g}$ & $0.1 \mathrm{~b}$ & $0.3 \mathrm{~b}$ & $0.3 \mathrm{~b}$ & $1.0 \mathrm{~b}$ \\
\hline Silver-on-black & $20.3 \mathrm{a}$ & $21.0 \mathrm{e}$ & $35.7 \mathrm{~d}$ & $37.4 \mathrm{c}$ & $0.6 \mathrm{~b}$ & $0.1 \mathrm{~b}$ & $0.8 \mathrm{~b}$ & $4.0 \mathrm{~b}$ \\
\hline SRM-Red & $20.3 \mathrm{a}$ & $22.6 \mathrm{a}$ & $37.3 \mathrm{c}$ & $35.9 \mathrm{~d}$ & $7.2 \mathrm{a}$ & $35.9 \mathrm{a}$ & $8.5 \mathrm{a}$ & $37.6 \mathrm{a}$ \\
\hline White-on-black & $19.2 \mathrm{~d}$ & $20.7 \mathrm{f}$ & $30.0 \mathrm{f}$ & $32.3 \mathrm{f}$ & $0.9 \mathrm{~b}$ & $4.4 \mathrm{~b}$ & $1.0 \mathrm{~b}$ & $5.3 \mathrm{~b}$ \\
\hline Yellow $^{\mathrm{v}}$ & $19.6 \mathrm{c}$ & $21.1 \mathrm{e}$ & $33.1 \mathrm{e}$ & $34.0 \mathrm{e}$ & $6.1 \mathrm{a}$ & $26.8 \mathrm{a}$ & $7.0 \mathrm{a}$ & $2.0 \mathrm{~b}$ \\
\hline
\end{tabular}

${ }^{\mathrm{z}}$ Average minimum and maximum soil temperature measurements at 5-cm depth were recorded daily for $49 \mathrm{~d}$ each year (19 Apr. to 6 June 1997 ; 14 Apr. to 2 June 1998) without replication.

${ }^{y}$ Cucumber beetle data were pooled over years as there were no significant interactions with years.

${ }^{\mathrm{x}} \mathrm{Al}-\mathrm{Or}=$ aluminum-organic, IRT-100 $=$ infrared transmitting, PST $=$ photosynthetic reduced transmitting, and SRM $=$ selective reflective mulch .

"Soil temperature and cucumber beetle mean separation within columns by Fisher's protected LSD test $(P \leq 0.05)$.

'Yellow-painted black mulch in 1997 and yellow-on-brown mulch (Polywest) in 1998. 
ences in 'Sangria' vine length at 4 weeks after transplanting due to mulch treatment.

Longer vines of watermelon plants grown on plastic mulches than bare ground agree with data reported by others (Soltani et al., 1995) evaluating black and white plastic mulches compared to bare ground. Soil temperatures (Table 2) may have contributed to differences in vine growth between plastic mulches and the bare-ground control as all plastic mulches had higher minimum and maximum (except silver-reflective in 1998) soil temperatures. Researchers using controlled environments have reported stem elongation in young watermelon plants treated at the end of the day with high FR/
R light (Decoteau and Friend, 1991; Decoteau and Hatt, 1997; Hatt and Decoteau, 1997). Our field results suggest that an altered plant microenvironment resulting from colored mulches affected the growth of watermelon main vine length. Three weeks after transplanting, watermelon main vines were growing out from the plastic surface and into the row middles. This could account for the reduced response of watermelon plants to plastic mulches 4 weeks after transplanting. Full canopy coverage of the plastic mulch treatments was achieved at 7 weeks after transplanting.

First and total harvest. First harvest of 'Honeyheart' was higher on black, blue- painted, IRT-100, and PST plastic mulches than on bare ground (Table 4). The first harvest yield was not significantly different among mulches. At first harvest, 'Honeyheart' fruit number per hectare was greater from mulched than bare ground plots with the exception of silver-reflective and white-on-black plastic mulches. There were no differences in fruit number among the mulch treatments. The late harvest (second and third harvests) was not affected by mulch treatments (data not presented). Total 'Honeyheart' (three harvests) yield and total fruit number were higher from mulched plots than bare ground plots, except for the PST and yellow plastic mulches. No

Table 3. Effect of colored plastic mulches on main vine length (m) in 'Honeyheart' and 'Sangria'watermelon in 1997 and 1998.

\begin{tabular}{|c|c|c|c|c|c|c|c|c|}
\hline \multirow[b]{4}{*}{ Treatment } & \multicolumn{4}{|c|}{1997} & \multicolumn{4}{|c|}{1998} \\
\hline & \multicolumn{2}{|c|}{ Honeyheart } & \multicolumn{2}{|c|}{ Sangria } & \multicolumn{2}{|c|}{ Honeyheart } & \multicolumn{2}{|c|}{ Sangria } \\
\hline & \multicolumn{8}{|c|}{ Weeks after transplanting } \\
\hline & 3 & 4 & 3 & 4 & 2 & 4 & 2 & 4 \\
\hline Al-Or brown ${ }^{2}$ & $0.82 \mathrm{~b}^{y}$ & $1.64 \mathrm{a}$ & $0.76 \mathrm{ab}$ & $1.49 \mathrm{a}$ & $0.19 \mathrm{a}$ & $1.45 \mathrm{ab}$ & $0.25 \mathrm{a}$ & $1.69 \mathrm{a}$ \\
\hline Bare ground & $0.37 \mathrm{~d}$ & $0.87 \mathrm{~b}$ & $0.34 \mathrm{c}$ & $0.63 \mathrm{~b}$ & $0.07 \mathrm{~b}$ & $0.83 \mathrm{c}$ & $0.11 \mathrm{~b}$ & $0.89 \mathrm{~b}$ \\
\hline Black & $1.00 \mathrm{a}$ & $1.83 \mathrm{a}$ & $0.82 \mathrm{a}$ & $1.82 \mathrm{a}$ & $0.14 \mathrm{a}$ & $1.19 \mathrm{~b}$ & $0.26 \mathrm{a}$ & $1.74 \mathrm{a}$ \\
\hline Blue-painted & $0.84 \mathrm{ab}$ & $1.69 \mathrm{a}$ & $0.88 \mathrm{a}$ & $1.87 \mathrm{a}$ & $0.15 \mathrm{a}$ & $1.45 \mathrm{ab}$ & $0.25 \mathrm{a}$ & $1.74 \mathrm{a}$ \\
\hline IRT-100 & $0.84 \mathrm{ab}$ & $2.01 \mathrm{a}$ & $0.89 \mathrm{a}$ & $1.95 \mathrm{a}$ & $0.18 \mathrm{a}$ & $1.48 \mathrm{ab}$ & $0.26 \mathrm{a}$ & $1.78 \mathrm{a}$ \\
\hline PST & $0.84 \mathrm{ab}$ & $1.60 \mathrm{a}$ & $0.97 \mathrm{a}$ & $1.91 \mathrm{a}$ & $0.16 \mathrm{a}$ & $1.43 \mathrm{ab}$ & $0.23 \mathrm{a}$ & $1.71 \mathrm{a}$ \\
\hline Silver-reflective & $0.81 \mathrm{~b}$ & $1.62 \mathrm{a}$ & $0.89 \mathrm{a}$ & $1.73 \mathrm{a}$ & $0.12 \mathrm{ab}$ & $1.52 \mathrm{a}$ & $0.16 \mathrm{ab}$ & $1.72 \mathrm{a}$ \\
\hline Silver-on-black & $0.85 \mathrm{ab}$ & $1.73 \mathrm{a}$ & $0.89 \mathrm{a}$ & $1.86 \mathrm{a}$ & $0.12 \mathrm{ab}$ & $1.54 \mathrm{a}$ & $0.22 \mathrm{ab}$ & $1.66 \mathrm{a}$ \\
\hline SRM-Red & $0.81 \mathrm{bc}$ & $1.72 \mathrm{a}$ & $0.91 \mathrm{a}$ & $1.91 \mathrm{a}$ & $0.18 \mathrm{a}$ & $1.69 \mathrm{a}$ & $0.23 \mathrm{a}$ & $1.66 \mathrm{a}$ \\
\hline White-on-black & $0.65 \mathrm{c}$ & $1.32 \mathrm{ab}$ & $0.71 \mathrm{ab}$ & $1.55 \mathrm{a}$ & $0.10 \mathrm{ab}$ & $1.28 \mathrm{ab}$ & $0.21 \mathrm{ab}$ & $1.53 \mathrm{a}$ \\
\hline Yellow $^{\mathrm{x}}$ & $0.70 \mathrm{bc}$ & $1.29 \mathrm{ab}$ & $0.63 \mathrm{~b}$ & $1.60 \mathrm{a}$ & $0.10 \mathrm{ab}$ & $1.25 \mathrm{ab}$ & $0.18 \mathrm{ab}$ & $1.63 \mathrm{a}$ \\
\hline
\end{tabular}

${ }^{\mathrm{z}} \mathrm{Al}$-Or $=$ aluminum-organic, IRT-100 = infrared transmitting, PST = photosynthetic reduced transmitting, and SRM = selective reflective mulch.

${ }^{y}$ Mean separation within columns by Fisher's protected LSD test $(P \leq 0.05)$.

${ }^{x}$ Yellow-painted black mulch in 1997 and yellow-on-brown (Polywest) in 1998.

Table 4. Effect of colored plastic mulches on marketable yield and culls in 'Honeyheart' watermelon, pooled over both years (1997 and 1998).

\begin{tabular}{|c|c|c|c|c|c|}
\hline \multirow[b]{2}{*}{ Treatment } & \multicolumn{2}{|c|}{ First harvest $^{\mathrm{z}}$} & \multicolumn{2}{|c|}{ Total harvest $^{z}$} & \multirow{2}{*}{$\begin{array}{c}\text { Cull } \\
\text { ton } \cdot \text { ha }^{-1}\end{array}$} \\
\hline & $\mathrm{t} \cdot \mathrm{ha}^{-1}$ & No. fruit/100ha & $\mathrm{t} \cdot \mathrm{ha}^{-1}$ & No fruit/100 ha & \\
\hline$\overline{\mathrm{Al}-O r}$ brown ${ }^{y}$ & $17.2 a b^{x}$ & $27.4 \mathrm{a}$ & $55.2 \mathrm{a}$ & $90.4 \mathrm{a}$ & $5.2 \mathrm{a}$ \\
\hline Bare ground & $2.8 \mathrm{~b}$ & $5.8 \mathrm{~b}$ & $26.4 \mathrm{~b}$ & $48.2 \mathrm{~b}$ & $10.0 \mathrm{a}$ \\
\hline Black & $27.2 \mathrm{a}$ & $45.8 \mathrm{a}$ & $61.0 \mathrm{a}$ & $103.6 \mathrm{a}$ & $7.0 \mathrm{ab}$ \\
\hline Blue-painted & $27.6 \mathrm{a}$ & $45.8 \mathrm{a}$ & $64.6 \mathrm{a}$ & $104.8 \mathrm{a}$ & $5.4 \mathrm{ab}$ \\
\hline IRT-100 & $23.0 \mathrm{a}$ & $36.6 \mathrm{a}$ & $60.8 \mathrm{a}$ & $99.0 \mathrm{a}$ & $4.6 \mathrm{ab}$ \\
\hline PST & $22.4 \mathrm{a}$ & $37.8 \mathrm{a}$ & $48.4 \mathrm{ab}$ & $79.4 \mathrm{a}$ & $10.2 \mathrm{a}$ \\
\hline Silver-reflective & $15.4 \mathrm{ab}$ & $23.6 \mathrm{ab}$ & $61.0 \mathrm{a}$ & $99.0 \mathrm{a}$ & $5.8 \mathrm{ab}$ \\
\hline Silver-on-black & $18.8 \mathrm{ab}$ & $31.4 \mathrm{a}$ & $61.6 \mathrm{a}$ & $102.2 \mathrm{a}$ & $8.0 \mathrm{a}$ \\
\hline SRM-Red & $18.0 \mathrm{ab}$ & $28.8 \mathrm{a}$ & $54.6 \mathrm{a}$ & $89.6 \mathrm{a}$ & $5.8 \mathrm{ab}$ \\
\hline White-on-black & $15.4 \mathrm{ab}$ & $23.6 \mathrm{ab}$ & $52.0 \mathrm{a}$ & $82.4 \mathrm{a}$ & $8.2 \mathrm{a}$ \\
\hline Yellow w & $19.8 \mathrm{ab}$ & $29.8 \mathrm{a}$ & $49.8 \mathrm{ab}$ & $78.2 \mathrm{a}$ & $3.6 \mathrm{~b}$ \\
\hline
\end{tabular}

${ }^{2}$ First harvest, 7 July 1997 and 22 June 1998, considered early marketable yield, the three harvests combined for total harvest (marketable) yield.

${ }^{\mathrm{y}} \mathrm{Al}-\mathrm{Or}=$ aluminum-organic, IRT-100 = infrared transmitting, PST $=$ photosynthetic reduced transmitting, and SRM = selective reflective mulch.

${ }^{x}$ Mean separation within columns by Fisher's protected LSD test $(P \leq 0.05)$.

wellow-painted black mulch in 1997 and yellow on brown mulch (Polywest) in 1998.

Table 5. Effect of colored plastic mulches on marketable yield and culls in "Sangria" watermelon, pooled over both years (1997 and 1998).

\begin{tabular}{|c|c|c|c|c|c|}
\hline \multirow[b]{2}{*}{ Treatment } & \multicolumn{2}{|c|}{ First harvest $^{z}$} & \multicolumn{2}{|c|}{ Total harvest $^{\mathrm{z}}$} & \multirow{2}{*}{$\begin{array}{c}\text { Cull } \\
\left(\mathrm{t} \cdot \mathrm{ha}^{-1}\right)\end{array}$} \\
\hline & $\mathrm{t} \cdot \mathrm{ha}^{-1}$ & No. fruit/100 ha & $\mathrm{t} \cdot \mathrm{ha}^{-1}$ & No fruit/100 ha & \\
\hline$\overline{\mathrm{Al}-O r}$ brown ${ }^{y}$ & $22.3{b c^{x}}^{x}$ & $20.2 \mathrm{bc}$ & $61.1 \mathrm{~b}$ & $61.1 \mathrm{~b}$ & $1.2 \mathrm{ab}$ \\
\hline Bare ground & $0.0 \mathrm{~d}$ & $0.0 \mathrm{~d}$ & $36.3 \mathrm{c}$ & $38.0 \mathrm{c}$ & $2.4 \mathrm{a}$ \\
\hline Black & $26.6 \mathrm{bc}$ & $25.4 \mathrm{bc}$ & $69.8 \mathrm{ab}$ & $68.5 \mathrm{~b}$ & $1.9 \mathrm{ab}$ \\
\hline Blue-painted & $23.4 \mathrm{bc}$ & $21.3 \mathrm{bc}$ & $66.9 \mathrm{ab}$ & $62.8 \mathrm{~b}$ & $0.2 \mathrm{~b}$ \\
\hline IRT-100 & $40.4 \mathrm{a}$ & $37.1 \mathrm{a}$ & $74.4 \mathrm{a}$ & $71.8 \mathrm{ab}$ & $1.5 \mathrm{ab}$ \\
\hline PST & $37.1 \mathrm{ab}$ & $34.2 \mathrm{ab}$ & $75.9 \mathrm{a}$ & $74.0 \mathrm{ab}$ & $0.3 \mathrm{~b}$ \\
\hline Silver-reflective & $26.3 \mathrm{bc}$ & $23.0 \mathrm{bc}$ & $67.8 \mathrm{ab}$ & $65.0 \mathrm{~b}$ & $0.8 \mathrm{ab}$ \\
\hline Silver-on-black & $29.5 \mathrm{ab}$ & $27.1 \mathrm{ab}$ & $85.0 \mathrm{a}$ & $83.4 \mathrm{a}$ & $2.2 \mathrm{a}$ \\
\hline SRM-Red & $20.1 \mathrm{c}$ & $19.1 \mathrm{c}$ & $66.1 \mathrm{ab}$ & $66.7 \mathrm{~b}$ & $2.0 \mathrm{ab}$ \\
\hline White-on-black & $22.2 \mathrm{bc}$ & $20.2 \mathrm{bc}$ & $65.3 \mathrm{ab}$ & $66.7 \mathrm{~b}$ & $1.0 \mathrm{ab}$ \\
\hline Yelloww & $27.5 \mathrm{bc}$ & $24.7 \mathrm{bc}$ & $65.0 \mathrm{ab}$ & $62.8 \mathrm{~b}$ & $1.7 \mathrm{ab}$ \\
\hline
\end{tabular}

${ }^{2}$ First harvest, 7 July 1997 and 22 June 1998, considered early marketable yield, the three harvests combined for total harvest (marketable yield).

${ }^{y} \mathrm{Al}-\mathrm{Or}=$ aluminum-organic, IRT-100 = infrared transmitting, PST = photosynthetic reduced transmitting, and SRM = selective reflective mulch.

${ }^{x}$ Mean separation within columns by Fisher's protected LSD test $(P \leq 0.05)$.

wellow-painted black mulch in 1997 and yellow on brown mulch (Polywest) in 1998. 
differences in total harvest were found among plastic mulches.

All plastic mulch treatments resulted in higher first 'Sangria' harvest, compared with the bare ground treatment (Table 5). The IRT100 mulch had the highest number of harvestable fruit per hectare and consequently among the greatest 'Sangria' first harvest yield, similar toPST and silver-on-black plastic mulches. The lowest yield (weight and number of fruit) were from SRM-Red, but yields were different only from the IRT-100, PST, and the silver-on-black mulches. Soil temperatures (Table 2) may have contributed to higher early yields from plastic mulches compared to bare ground. All the mulches had higher minimum soil temperatures than bare ground. In addition, soil temperatures may have influenced the differences in early yield of darker (IRT-100) compared to the lighter mulches (white-on-black; yellow). IRT100 had the highest maximum soil temperature while white-on-black and yellow mulch had the lowest soil temperatures of all mulches. The late harvests (second and third harvests) were not affected by mulch treatments (data not presented).

Plastic mulches had higher total yield than the bare ground treatment. Higher watermelon yields from black and/or white plastic mulches compared to bare ground is in general agreement with previous watermelon research (Brinen et al., 1979; Sanders et al., 1999; Soltani et al., 1995). Among plastic mulch treatments, silver-on-black, PST, and IRT-100 mulch had a higher total harvest yield than Al-Or brown mulch, which was among the lowest yielding mulch treatments for 'Sangria' total harvest. Plastic mulch treatments for 'Sangria' had higher total harvest fruit number than bare ground. Among plastic mulch treatments, silver-on-black mulch produced among the highest fruit number; PST and IRT-100, however, had similar results. Average fruit weight of both cultivars was not different among mulch treatments, but was lowest on bare ground (data not presented). There were no significant interactions of cultivars and mulch treatments in our study for beetle populations and plant growth (results not presented). Cultivar and mulch treatment interactions, however, were significant for first harvest weight and number of fruit, and total harvest weight.

Culls. Plastic mulch treatments affected cull weight at total harvest (Tables 4 and 5).
There were differences between cultivars and the interaction between mulch treatment and cultivars was significant (results not shown). The cull weight for 'Honeyheart' was higher than for 'Sangria.' There was a higher amount of 'Honeyheart' cull fruit in the PST, bare ground, white-on-black and silver-on-black mulch treatments than the yellow mulch treatment (Table 4); the bare ground and silver-on-black mulch had more 'Sangria' cull fruit when compared with PST and blue-painted plastic mulch treatments (Table 5).

Fruit length, diameter, and soluble solids. Fruit length and soluble solids of both cultivars were not affected by mulch treatments (data not presented). For total harvest, 'Honeyheart' fruit diameter in bare ground plots was lower than fruit from plastic mulch treatments, while silver-reflective, blue-painted, and PST plastic mulch treatments had higher 'Sangria' fruit diameter than the bare ground treatment (data not presented).

In summary, the silver reflective plastic mulch had the lowest and SRM-Red and yellow plastic treatments the highest cucumber beetle populations, but results showed no statistical difference from other plastic treatments. No effect was observed between plastic mulch treatments on field grown watermelon main vine length after four week after transplanting. Plastic mulches affected yield of 'Honeyheart' and 'Sangria' watermelon differently. No difference among mulch treatments was found for the first or total 'Honeyheart' harvest. IRT100, PST, and silver-on-black plastic mulch treatments, however, had higher first and total 'Sangria' yield when compared to the other plastic mulches. Higher 'Sangria' first harvest was a result of higher numbers of first fruit produced. In addition, plastic mulch treatments did not affect average fruit weight, fruit total soluble solids, and fruit length and diameter. Further research is required to investigate the influence of colored mulches on watermelon growth and yield.

\section{Literature Cited}

Boudreaux, J.E. 1996. Commercial vegetable recommendations. La. Coop. Ext. Serv. Publ. 2433.

Brinen, G.H., S.J. Locascio, and G.W. Elmstrom. 1979. Plant and row spacing, mulch, and fertilizer rate effects on watermelon production. J. Amer. Soc. Hort. Sci. 104:724-726.

Brown, J., J. Dangler, F. Woods, and M. Ken. 1993. Delay in mosaic virus onset and aphid vector reduction in summer squash grown on reflective mulches. HortScience 28:895-896.

Decoteau, D. and F. Friend. 1991. Phytochromeregulated growth of young watermelon plants. J. Amer. Soc. Hort. Sci. 116:512-515.

Decoteau, D. and H. Hatt. 1997. Day-long alterations of the photomorphogenic light environment affect young watermelon plant grow: Implications for use with rowcovers. HortTechnology 7:261-263.

Decoteau, D., M. Kasperbauer, and P. Hunt. 1989. Mulch surface color affects yield of freshmarket tomatoes. J. Amer. Soc. Hort. Sci. 114: 216-219.

Decoteau, D., M. Kasperbauer, and P. Hunt. 1990. Bell pepper plant development over mulches of diverse colors. HortScience 25:460-462.

Hatt, H. and D. Decoteau. 1997. Sensitivity of shoots and roots of young watermelon plants to endof-day red and far-red light. J. Amer. Soc. Hort. Sci. 122:481-484.

Hochmuth, G. 1992. Fertilizer management for watermelon, p. 11-14 In: D.N. Maynard (ed.). Watermelon production guide for Florida. Fla. Coop. Ext. SP 113.

Hunt, P., T. Mathey, and M. Kasperbauer. 1990. Cowpea yield response to light reflected from different colored mulches. Crop Sci. 30:1292-1294.

Lamont, W., K. Sorensen, and C. Averre. 1990. Painting aluminum strips on black plastic mulch reduces mosaic symptoms on summer squash. HortScience 25:1305.

Matheny T., P. Hunt, and M. Kasperbauer. 1992. Potato tuber production in response to reflected light from different colored mulches. Crop Sci. 32:1021-1024.

Sanders, D.C., J.D. Cure, and J.R. Schultheis. 1999. Yield response of watermelon to planting density, planting pattern, and polyethylene mulch. HortScience 34:1221-1223.

Schalk, J.M., C.S. Creighton, R.L. Fery, W.R. Sitterly, B.W. Davis, T.L. McFadden, and A. Day. 1979. Reflective film mulches influences insect control and yield in vegetables. J. Amer. Soc. Hort. Sci. 104:759-762.

Schalk, J.M. and M. LeRon Robbins. 1987. Reflective mulches influence plant survival, production, and insect control in fall tomatoes. HortScience 22:30-32

Soltani, N., J. LaMar, and A. Hamson. 1995. Growth analysis of watermelon plants grown with mulches and rowcovers. J. Amer. Soc. Hort. Sci. 120:1001-1009.

Taber, H. 1993. Early muskmelon production with wavelength-selective and clear plastic mulches. HortTechnology 3:78-80.

Zalom, F.G. and W.S. Cranshaw. 1981. Effects of aluminum foil mulch on parasitism and fecunduti of apterous Myzus persicae (Homoptera: Aphididae). Great Lakes Entomol. 14:171-176. 